\title{
The Research on the Design of the Resource-Sharing Course of the basic Pattern on the Network Platform
}

\author{
Qiongmei Xie
}

Pingxiang University, Pingxiang, Jiangxi, 337000

Key words: network platform, resource sharing, curriculum design

\begin{abstract}
The article reviewed the curriculum construction of the resource-sharing course of the Basis Pattern as the example, focusing on the idea and the process of the course by illustrating its resource analysis, structural design and the style of interface design in order to build a network of resource sharing for improving the teaching quality.
\end{abstract}

\section{Introduction}

The construction of the resource-sharing course grows and improves together with the rapid development of the open education, which is the product of the informatization. Supported by the abundant media resource, the open education has enrich the teaching methods, motivated the study enthusiasm and interest of the students, cultivated their innovative ideas and abilities by channeling resources, like pictures, animations, music, and videos etc. into the class. The network platform has satisfied the teachers' requirements of designing the study sections, the curriculum outline and the teaching methods, and meanwhile deepening the teacher-student interaction. A well-established resource-sharing network is the core of the open education, and is an important guarantee for a smooth teaching activity. The resource-sharing course is a combination of high-quality, shared resource and the sharing activities. The high quality is the basis of the course, which takes the transformation as the key, and aims to share.[1] Directed by the transformation of curriculum resources as a part of the National Quality Open Course-ware Project, the construction of the resource-sharing courses should highlight its features of abundant teaching resource, diversified narration styles, extensive function of self-learning and the close interaction between the teacher and the students.

\section{The resource analysis of the Basic Pattern}

The actual needs of teaching should be considered in the curriculum design on the network platform so as to serve for the aims and demands of the teaching. And the courses should be conducted from the basic premise of the systematicness and integrity of the curriculum resources, as the full sharing activity be adopted as its elementary object, emphasizing the universality, characteristic and the usability of the curriculum resource. As a provincial-level curriculum of resource sharing, the Basic Pattern, based on the demands of the teaching implementation resource sharing and the interactive seminar, has developed a network platform of teaching, on which the introduction of the curriculum, practical teaching, teacher-student interaction, classroom features, teaching resource library and some excellent works are demonstrated to support the functions of learning resource sharing, teaching interaction, synchronous teaching and so on.

According to the Technical Requirements of the Construction of the National Resource-sharing 
Courses, the basic resource must be organize in the light of the course summary, teaching units and the teaching resource, and add the curriculum module if necessary. The basic resource of the resource-sharing course of the Basic Pattern in the network platform construction reflected mainly in three aspects: the curriculum introduction, the teaching units, and the teaching resource library. [2]The curriculum introduction consists of the curriculum program, the teaching outline, the evaluation system, the team of teaching, the curriculum schedule. The teaching units include: Chapter one--the previous and the present of pattern; Chapter two--the integrity of pattern; Chapter three--the eternal beauty of the pattern; Chapter four--the charm of pattern; Chapter five--the flexibility of pattern; Chapter six--practice. The teaching resource library includes the Classical Patterns, the Integrated Vectors and the Foreign Classical Patterns.

Additional resource is a relatively available diversified and ancillary resource, which is applied in the teaching and learning links to support the process of course teaching. The resource development of the resource-sharing course mainly aims to assist and deepen the teaching. Most of the resources are shared in forms of the curriculum cases, the teacher-student interaction and the excellent works. The interface of the net work platform shall be pleasing, concise, and easy to operate. The resource-sharing course, mainly embodies its additional resources in the practical teaching, the teacher-student interaction, the course features and the outstanding works. The practical teaching includes the content of the course with a brief literal explanation, the record of practice, the practical activities and the projects of practice. In the interface of teacher-student interaction, columns of Leave Messages and Ask Questions are set for the students to ask for the information they need.

\section{The structural design of the Basic Pattern}

In the development of the resource-sharing course of the Basic Pattern, the first concern is to choose a suitable textbook, analyze the content, design the frame of the resource and develop the specific content according to the structure of the textbook.

The functions of the textbook can be concluded into two aspects: First, it can be the resource for students to learn the course. Second, it is a tool in term of students' study. As the major learning resource in students' curriculum learning, textbook should strive to compact and generalize the accumulated knowledge of our long-term practical experience, and conduct it in a way that is acceptable and understandable for the learners as an important material to learn the relevant courses and professional skills, providing the necessary methods and approaches. Basic Pattern, as a resource-sharing course, was compiled by the lecturer and the teaching team. It has been constantly revised and improved after years of experience-accumulation and practicing of the team. [3]The content of the textbook has been continually updated to enhance the students' interest of self-learning by adding the new teaching content and the practical teaching cases etc. And the features of the resource-sharing course have been fully considered when compiling the textbook. Supported by its universality, practicability and characteristics, the textbook was compiled to serve for the teachers and students of the college and the social learners, which meets the constructive requirements of the recourse-sharing course of the Basic Pattern.

C. Reigeluth, the American educational technologist, proposed an instructional design theory of "elaborate processing”, based on the previous research. This theory not only used the level analysis of the knowledge structure but also involved the in-depth learning and the design of the teaching content sequence in the cognitive progress, emphasizing the integrity and utilization of the various teaching strategic methods and the whole cognition of the knowledge. It takes both the learners' psychological order and the logical system of the subject into the consideration at the same time, 
and provides the operable guiding theory for the teachers and the students According to the theory of elaborate processing, the content of the textbook has been detailed and listed as knowledge points. This kind of design covers all the unites as that the students can find their knowledge points step by step to consolidate their learning easily.

The construction of the Basic Pattern has shifted from the development of the static resources, such like the website and the learning course-ware etc. to the dynamic resource development, covering many aspects such like the various learning activities, the course teaching and the study evaluation etc. It focuses not only on the media present of the curriculum content, but primarily on the designs that reflect the teaching strategies and the learning activities. And it meet the requirements of the three-dimensional instructional resources, which is formed by the teaching content, teaching process and teaching evaluation.[4]For example, the courses practice record of 2012 to 2016 can be found on the network platform. Click on the title of Practical Teaching in Wenquan, Mashan in September, 2012, we can see that the college has organized the students to watch the nuo dance in a temple in Wenquan. (Nuo , a kind of folk exorcism activity of China) Wei Yuanhui the folk art expert of Pingxiang and Peng Anbao the museum director had been invited to introduce the nuo culture to the students who were later led to visit the 90-odd-year-old dancer of the Nuo ceremony, and understand the current situation of the nuo culture in Pingxiang, including the dance, temples and masks of the nuo, which helped the students to gain a better and wider knowledge over the patterns of the local culture. Click on the Back button, the record of the practical teaching will be listed, including the time, place, participants and pictures etc. Under the structure of the Basic Pattern, learners can fully bring up their dependency, which makes the teaching no longer a teaching or being-taught process. The multiform learning resource allows the learners to study freely, provides them a platform for communication, and gives them the personalized guide and assistance over the study.

\section{The interfacial design of the Basic Pattern}

The interfacial design is an important component in the construction of the resource-sharing course. The design of the interface not only affects the design of the curriculum resource structure but also affects the guide and the maintenance of the students' interest. Therefore, the interface must be concise, distinctive, user-friendly and appropriately separated. The interface of Basic Pattern was designed by a professional design team. And the online teaching platform of this course has won the second prize of the 15th National Multimedia Course-ware( College of Arts) for its concise layout, elegant design of the overall style and the visual sense of beauty that affects the learners.

As a concerted product work of the team, interfacial design requires the joint efforts of the professional teachers, website designers and programmers as well. However, due to the different personalities and views of the team members, communication and joint planning must carried out before the designing. First, teachers provide the resource content, formulate a general structure of the website, and then prioritize the columns that lead to the content, together with the designer. According to the website structure, the designer will choose a suitable site-building mode for the link steps of the website so as to implement the interfacial functions, improve the interfacial operation, and complete the interfacial design of the website.

The Specifications for Evaluating Web-based Courses(CELTS-22) demands the online course to the "unified in interfacial style, easy to operate, concise in the content, and relatively perfect in the functions". Therefore, the layout, color and web-navigation should be decided ahead. Emphasizing the overall visual process, creating a comfortable visual environment, and simplifying the operative functions are also necessary. First, the concise interfacial design of the course and the exquisite 
background pictures, columns and buttons that related to the content demonstrate a beautiful and vivid visual effect. The backbone of the course is just clear, as the course introduction, teaching units, practical teaching, teacher-student interaction, course features and excellent works are posted on the interface of the web, downwards the teaching resource library of the classical patterns, integrated vectors and the foreign classical patterns. Click on the course introduction comes along a new interface which is a combination of the multimedia objects, such like animation, pictures, words and videos etc. The arrangement is of great importance. The visual process should be considered as the first issue, increase the appeal to the students by putting the words on the "best visual field". On the basis of the functions and the prioritization of the content so as to make the website easier for the students to operate on the platform. The color of the interface should be in accordance with the visual laws of the chromatics, emphasizing the sense of depth contrast and the harmony of the colors. The distribution of the color should highlight the keynote, present a delicate quality level, and create a pleasing atmosphere of the colors. The phenomenon of so many colors are being used in a mess is inadvisable here. The navigation tab is the major icon of the interface. The design of the icon is actually the process of information classification, so it must be clear, accurate and unambiguous.

\section{Conclusion}

There are so many researches of the curriculum design over the resource-sharing courses of the notwork platform. However, not much attention has paid to the resource-sharing course of the Basic Pattern. It is well-timed and of great necessary to integrate the curriculum resource, and build a course platform so as to permeate the efforts into the real teaching activities, conforming to the trend of the informatization through the development and research of the interaction teaching mode of the teaching resource integrity. Basic Pattern is a basic course of artistic designing. The previous curriculum teaching mainly focuses on the classroom teaching and the practical teaching. Our college has conducted the study of the teaching reform on the course informatization, but research on the resource-sharing courses on the network platform has not be explored yet. And research of the resource-sharing course design would make a lot of sense for the study of the students and the professional development of the teachers in the art colleges.

\section{Acknowledgement}

Project fund: The phrased objectives of the Project of Jinagxi Higher Education Reform of the Model Study over the Interactive Teaching on the Resource-sharing Network Platform. (number:JXJG-15-22-4)

\section{References}

[1] Li Jianchun. The exploration and practice of the upgrade from the excellent courses to the excellent course-sharing courses---take the <Container Transportation Practice $>$ as the example[J]. Higher Education BBS.2013(8)

[2] Yuan Zhijian, He Wenjuan. The comparison between the excellent courses and the excellent course-sharing courses [J]. China Education Technique \& Equipment,2013(12)

[3] Department of the Higher Education, Ministry of Education of the P. R. China, the technical requirements of the resource-sharing courses[OL]. Version 2013

[4] Li Xingbao, Wu Xiying. The process model and value orientation of the knowledge-sharing 
community of the teachers. E-Education Research,2013(3):73-80 